(c) 2001 International Press

Adv. Theor. Math. Phys. 5 (2001)

\title{
Nonlinear differential equations
}

\section{for the correlation functions of the 2D Ising model on the cylinder}

\author{
Oleg Lisovyy \\ Bogolyubov Institute for Theoretical Physics \\ Metrolohichna str., 14-b, Kyiv-143, 03143, Ukraine \\ lisovy@ukr.net
}

\begin{abstract}
We derive determinant representations and nonlinear differential equations for the scaled 2-point functions of the 2D Ising model on the cylinder. These equations generalize well-known results for the infinite lattice (Painlevé III equation and the equation for the $\tau$-function of Painlevé V).
\end{abstract}

e-print archive: http://xxx.lanl.gov/hep-th/0108015 


\section{Introduction}

One of the most beautiful results of the modern mathematical physics is the connection between correlations in the 2D Ising model and Painlevé functions. Quantum field theory and condensed matter physics usually deal with Dyson equations for the Green functions. These equations, however, can not be reduced to closed form, i. e. to evaluate a propagator from Dyson equations one must first perturbatively calculate the vertex. The Ising model provides a remarkable example of closed differential equations for the correlation functions and, moreover, these equations prove to be integrable.

Scaled 2-point function of the 2D Ising model on the infinite lattice

$$
\begin{aligned}
& \left\langle\sigma(0,0) \sigma\left(r_{x}, r_{y}\right)\right\rangle^{(-)}=\xi \tau_{-}(t), \quad s>1 \\
& \left\langle\sigma(0,0) \sigma\left(r_{x}, r_{y}\right)\right\rangle^{(+)}=\xi \tau_{+}(t), \quad s<1
\end{aligned}
$$

can be expressed through the functions $\tau_{ \pm}(t)$ of the single parameter

$$
t=\frac{|s-1|}{\sqrt{2}} \sqrt{r_{x}^{2}+r_{y}^{2}}
$$

where

$$
s=\sinh 2 \mathcal{K}, \quad \xi=\left|1-s^{-4}\right|^{\frac{1}{4}}
$$

and $\mathcal{K}$ is the Ising coupling constant.

As it was shown in $[1,2]$, the function

$$
\eta(\theta)=\frac{\tau_{-}(2 \theta)-\tau_{+}(2 \theta)}{\tau_{-}(2 \theta)+\tau_{+}(2 \theta)}
$$

satisfies the equation

$$
\eta^{\prime \prime}=\frac{1}{\eta}\left(\eta^{\prime}\right)^{2}-\frac{1}{\theta} \eta^{\prime}+\eta^{3}-\eta^{-1}
$$

and the asymptotical boundary conditions

$$
\eta(\theta) \sim 1-\frac{2}{\pi} K_{0}(2 \theta)
$$

at $\theta \rightarrow \infty$. The equation (1.6) is a particular case of Painlevé III equation. Furthermore, if we define

$$
\zeta(t)=t \frac{d \ln \tau_{ \pm}(t)}{d t}
$$


then in both cases [3]

$$
\left(t \zeta^{\prime \prime}\right)^{2}=4\left(t \zeta^{\prime}-\zeta\right)^{2}-4\left(\zeta^{\prime}\right)^{2}\left(t \zeta^{\prime}-\zeta\right)+\left(\zeta^{\prime}\right)^{2} .
$$

This is the equation for the $\tau$-function of Painlevé $V$. The most interesting feature of (1.9) is that it contains correlation functions $\tau_{ \pm}$themselves instead of their combination (1.5).

There are several similar results for the correlations in one-dimensional quantum models: impenetrable Bose gas [4], XY and XXZ spin chains [5, 6], sine-Gordon field model [7]. In two dimensions, there were no examples of this kind other than spin-spin correlation functions of the Ising model on the infinite plane. Quite recently $[8,9]$ the form factor representation for the correlation function was calculated for the Ising model on a cylinder. This gave an opportunity to evaluate the susceptibility and to investigate its singularity structure in the complex temperature plane in the case of a finite-size lattice. In the present paper, we continue the study of correlations in the Ising model on a cylinder.

In this case, the rotational invariance is broken even in the scaling limit and one has three scaling parameters

$$
x=\frac{|1-s|}{\sqrt{2}} r_{x}, \quad y=\frac{|1-s|}{\sqrt{2}} r_{y}, \quad \beta=\frac{|1-s|}{\sqrt{2}} N,
$$

where $N$ is the number of sites on the base of the cylinder. Correlation function in the scaling limit

$$
\begin{aligned}
& \left\langle\sigma(0,0) \sigma\left(r_{x}, r_{y}\right)\right\rangle^{(-)}=\xi \widetilde{\xi}_{T}(\beta) \mathrm{e}^{-|x| / \widetilde{\Lambda}(\beta)} \tau_{-}(x, y, \beta), \quad s>1, \\
& \left\langle\sigma(0,0) \sigma\left(r_{x}, r_{y}\right)\right\rangle^{(+)}=\xi \widetilde{\xi}_{T}(\beta) \mathrm{e}^{-|x| / \widetilde{\Lambda}(\beta)} \tau_{+}(x, y, \beta), \quad s<1 .
\end{aligned}
$$

The notation will be explained in the next sections. Before summarize main results of the paper, we define complex variables

$$
z=-\frac{|x|-i y}{2}, \quad \bar{z}=-\frac{|x|+i y}{2}
$$

and the function

$$
\varphi=\ln \frac{\tau_{-}+\tau_{+}}{\tau_{-}-\tau_{+}}
$$

It will be shown that $\varphi$ satisfies sinh-Gordon equation

$$
\varphi_{z \bar{z}}=\frac{1}{2} \sinh 2 \varphi
$$

Besides that, we will derive equations for $\tau_{ \pm}$separately. If we define

$$
u=\ln \tau_{ \pm},
$$


then $u$ satisfies the equation

$$
u_{z \bar{z}}\left(u_{z z \overline{z z}}+2 u_{z \bar{z}}^{2}-u_{z \bar{z}}\right)=u_{z z \bar{z}} u_{z \overline{z z}}-u_{z z} u_{\overline{z z}}
$$

in both cases. This is quite a fascinating result. In fact, we will find a general $N$-solitonic solution of (1.14) and the Ising scaled correlation function is infinite-solitonic one. As far as the author is aware, the equation (1.14) is not one of common knowledge. Nevertheless, it seems to be integrable and complete investigation of it (construction of the Lax representation, hamiltonian structure, etc.) is a challenging task.

This paper is organized as follows. The scaling limit of the formulae of $[8$, $9]$ and determinant representations for the correlation functions on a cylinder are obtained in Section 2. On the infinite lattice correlation functions can be expressed through Fredholm determinants of integral operators and on the cylinder - through the determinants of some infinite dimensional matrices. The derivation of differential equations (1.12), (1.14) and a brief rederivation of Painlevé III (1.6) and Painlevé V (1.9) equations are given in Section 3.

\section{Scaling limit and determinant representations for the correlation functions}

The most convenient representations for the investigation of correlation functions on the infinite lattice are the so-called form factor expansions $[10,11]$

$$
\begin{gathered}
\left\langle\sigma(0,0) \sigma\left(r_{x}, r_{y}\right)\right\rangle^{(-)}=\xi \sum_{n=0}^{\infty} g_{2 n}, \quad s>1 \\
\left\langle\sigma(0,0) \sigma\left(r_{x}, r_{y}\right)\right\rangle^{(+)}=\xi \sum_{n=0}^{\infty} g_{2 n+1}, \quad s<1 \\
g_{n}=\frac{1}{n !(2 \pi)^{n}} \int_{-\pi}^{\pi} \ldots \int_{-\pi}^{\pi} d q_{1} \ldots d q_{n} F_{n}^{2}[q] \prod_{j=1}^{n} \frac{\mathrm{e}^{-\left|r_{x}\right| \gamma\left(q_{j}\right)+i r_{y} q_{j}}}{\sinh \gamma\left(q_{j}\right)}, \quad g_{0}=1, \\
F_{n}[q]=\prod_{1 \leq i<j \leq n} \frac{\sin \frac{q_{i}-q_{j}}{2}}{\sinh \frac{\gamma\left(q_{i}\right)+\gamma\left(q_{j}\right)}{2}}, \quad F_{1}=1,
\end{gathered}
$$

where the function $\gamma(q)$ is defined by equation

$$
\cosh \gamma(q)=s+s^{-1}-\cos q
$$

and condition $\gamma(q)>0$. 
It proved $[8,9]$ that the correlation function on the cylinder with $N$ sites on the base may be written in similar form, namely

$$
\begin{gathered}
\left\langle\sigma(0,0) \sigma\left(r_{x}, r_{y}\right)\right\rangle^{(-)}=\left(\xi \cdot \xi_{T}\right) \mathrm{e}^{-\left|r_{x}\right| / \Lambda} \sum_{n=0}^{[N / 2]} g_{2 n}, \quad s>1 \\
\left\langle\sigma(0,0) \sigma\left(r_{x}, r_{y}\right)\right\rangle^{(+)}=\left(\xi \cdot \xi_{T}\right) \mathrm{e}^{-\left|r_{x}\right| / \Lambda} \sum_{n=0}^{[(N-1) / 2]} g_{2 n+1}, \quad s>1 \\
g_{n}=\frac{\mathrm{e}^{-n / \Lambda}}{n ! N^{n}} \sum_{[q]}^{(b)} F_{n}^{2}[q] \prod_{j=1}^{n} \frac{\mathrm{e}^{-\left|r_{x}\right| \gamma\left(q_{j}\right)+i r_{y} q_{j}-\nu\left(q_{j}\right)}}{\sinh \gamma\left(q_{j}\right)}, \quad g_{0}=1
\end{gathered}
$$

The main differences between (2.1)-(2.3) and (2.5)-(2.7) are the substitution of the integration by the summation over discrete bosonic spectrum of quasimomentum $\left(q_{j}=\frac{2 \pi}{N} k, k=0,1, \ldots, N-1\right)$ and the appearance of cylindrical parameters $\xi_{T}, \Lambda$ and $\nu(q)$

$$
\begin{gathered}
\ln \xi_{T}=\frac{N^{2}}{2 \pi^{2}} \int_{0}^{\pi} \frac{d p d q \gamma^{\prime}(p) \gamma^{\prime}(q)}{\sinh (N \gamma(p)) \sinh (N \gamma(q))} \ln \left|\frac{\sin ((p+q) / 2)}{\sin ((p-q) / 2)}\right|, \\
\Lambda^{-1}=\frac{1}{\pi} \int_{0}^{\pi} d p \ln \operatorname{coth}(N \gamma(p) / 2) \\
\nu(q)=\frac{1}{\pi} \int_{0}^{\pi} \frac{d p\left(\cos p-\mathrm{e}^{-\gamma(q)}\right)}{\cosh \gamma(q)-\cos p} \ln \operatorname{coth}(N \gamma(p) / 2) .
\end{gathered}
$$

The finite number of terms in the sums (2.5) and (2.6) provides an opportunity of independent check of the formulae $[8,9]$ for small $N$.

The scaling limit of the Ising model is of interest mainly due to its applications in quantum field theory. This limit implies that

$$
\begin{gathered}
\gamma(0) \rightarrow 0, \quad r_{x} \rightarrow \infty, \quad r_{y} \rightarrow \infty, \quad N \rightarrow \infty \\
\gamma(0) r_{x} \rightarrow x=\text { const }, \quad \gamma(0) r_{y} \rightarrow y=\text { const }, \quad \gamma(0) N \rightarrow \beta=\text { const. }
\end{gathered}
$$

In this case [12] the formulae (2.5)-(2.7) reduce to

$$
\left\langle\sigma(0,0) \sigma\left(r_{x}, r_{y}\right)\right\rangle^{(-)}=\xi \widetilde{\xi}_{T}(\beta) \mathrm{e}^{-|x| / \widetilde{\Lambda}(\beta)} \sum_{n=0}^{\infty} \widetilde{g}_{2 n}, \quad s>1,
$$




$$
\begin{gathered}
\left\langle\sigma(0,0) \sigma\left(r_{x}, r_{y}\right)\right\rangle^{(+)}=\xi \widetilde{\xi}_{T}(\beta) \mathrm{e}^{-|x| / \widetilde{\Lambda}(\beta)} \sum_{n=0}^{\infty} \widetilde{g}_{2 n+1}, \quad s<1, \\
\widetilde{g}_{n}=\frac{1}{n ! \beta^{n}} \sum_{[l]=-\infty}^{\infty} \widetilde{F}_{n}^{2}[l] \prod_{j=1}^{n} \frac{\mathrm{e}^{-|x| \sqrt{1+\left(\frac{2 \pi l_{j}}{\beta}\right)^{2}}+i y \frac{2 \pi l_{j}}{\beta}-\widetilde{\nu}\left(l_{j}, \beta\right)}}{\sqrt{1+\left(\frac{2 \pi l_{j}}{\beta}\right)^{2}}}, \quad \widetilde{g}_{0}=1, \\
\widetilde{F}_{n}[l]=\prod_{1 \leq i<j \leq n} \frac{\frac{2 \pi l_{i}}{\beta}-\frac{2 \pi l_{j}}{\beta}}{\sqrt{1+\left(\frac{2 \pi l_{i}}{\beta}\right)^{2}}+\sqrt{1+\left(\frac{2 \pi l_{j}}{\beta}\right)^{2}}} .
\end{gathered}
$$

The summation over $[l]$

$$
\sum_{[l]=-\infty}^{\infty}=\sum_{l_{1}=-\infty}^{\infty} \cdots \sum_{l_{n}=-\infty}^{\infty}
$$

is taken over integer values of $l_{j}$, and the quantities $\widetilde{\xi}_{T}(\beta), \widetilde{\Lambda}(\beta), \widetilde{\nu}(l, \beta)$ are defined by formulae

$$
\begin{gathered}
\ln \widetilde{\xi}_{T}(\beta)=\frac{\beta^{2}}{2 \pi^{2}} \int_{0}^{\infty} \int_{0}^{\infty} \frac{p d p q d q \ln \left|\frac{p+q}{p-q}\right|}{\sqrt{p^{2}+1} \sqrt{q^{2}+1} \sinh \left(\beta \sqrt{p^{2}+1}\right) \sinh \left(\beta \sqrt{q^{2}+1}\right)}, \\
\widetilde{\Lambda}^{-1}(\beta)=\frac{1}{\pi} \int_{0}^{\infty} d p \ln \operatorname{coth} \frac{\beta \sqrt{1+p^{2}}}{2} \\
\widetilde{\nu}(l, \beta)=\frac{2}{\pi} \int_{0}^{\infty} d p \frac{\sqrt{1+\left(\frac{2 \pi l}{\beta}\right)^{2}}}{1+\left(\frac{2 \pi l}{\beta}\right)^{2}+p^{2}} \ln \operatorname{coth} \frac{\beta \sqrt{1+p^{2}}}{2} .
\end{gathered}
$$

Let us denote

$$
u_{i}=\frac{2 \pi l_{i}}{\beta}+\sqrt{1+\left(\frac{2 \pi l_{i}}{\beta}\right)^{2}}, \quad u_{i}^{-1}=-\frac{2 \pi l_{i}}{\beta}+\sqrt{1+\left(\frac{2 \pi l_{i}}{\beta}\right)^{2}} .
$$

It is easily verified that

$$
\widetilde{F}_{n}^{2}[l]=\prod_{1 \leq i<j \leq n}\left(\frac{u_{i}-u_{j}}{u_{i}+u_{j}}\right)^{2}
$$


The right hand side of the last formula can be expressed through Vandermond determinant of a matrix with dimension $n \times n$ :

$$
\operatorname{det}_{n}\left\|\frac{1}{u_{i}+u_{j}}\right\|=\prod_{i=1}^{n}\left(\frac{1}{2 u_{i}}\right) \prod_{1 \leq i<j \leq n}\left(\frac{u_{i}-u_{j}}{u_{i}+u_{j}}\right)^{2} .
$$

If we make use of this fact and denote

$$
E_{i} \equiv E_{i}(\beta)=\left[\frac{2 u_{i} \mathrm{e}^{-\widetilde{\nu}\left(l_{i}, \beta\right)}}{\sqrt{1+\left(\frac{2 \pi l_{i}}{\beta}\right)^{2}}}\right]^{\frac{1}{2}},
$$

then for $\widetilde{g}_{n}$ we obtain

$$
\widetilde{g}_{n}=\frac{1}{n ! \beta^{n}} \sum_{[l]=-\infty}^{\infty} \operatorname{det}_{n}\left\|\frac{E_{i} E_{j} \exp \left[-\frac{|x|-i y}{2} \frac{u_{i}+u_{j}}{2}-\frac{|x|+i y}{2} \frac{u_{i}{ }^{-1}+u_{j}{ }^{-1}}{2}\right]}{u_{i}+u_{j}}\right\| .
$$

Let us consider an arbitrary square matrix $\widehat{K}$ with the elements $K_{m n}$ and construct the determinant

$$
\begin{aligned}
& \left|1+\frac{\widehat{K}}{\beta}\right|=\mathrm{e}^{\operatorname{Tr} \ln \left(1+\frac{\widehat{K}}{\beta}\right)}=\mathrm{e}^{\operatorname{Tr}\left(\frac{1}{\beta} \widehat{K}-\frac{1}{2 \beta^{2}} \widehat{K}^{2}+\ldots\right)}= \\
= & 1+\frac{1}{\beta} \operatorname{Tr} \widehat{K}+\frac{1}{2 \beta^{2}}\left[(\operatorname{Tr} \widehat{K})^{2}-\operatorname{Tr}\left(\widehat{K}^{2}\right)\right]+\ldots= \\
= & 1+\frac{1}{\beta} \sum_{m} K_{m m}+\frac{1}{2 \beta^{2}} \sum_{m, n}\left|\begin{array}{cc}
K_{m m} & K_{m n} \\
K_{n m} & K_{n n}
\end{array}\right|+\ldots
\end{aligned}
$$

as the expansion over $\frac{1}{\beta}$. After comparing these expressions with (2.16), it is easy to see that

$$
\begin{gathered}
\sum_{n=0}^{\infty} \widetilde{g}_{n}=\left|1+\frac{1}{\beta} \widehat{K}\right|, \\
\sum_{n=0}^{\infty}(-1)^{n} \widetilde{g}_{n}=\left|1-\frac{1}{\beta} \widehat{K}\right|,
\end{gathered}
$$

where the elements of the infinite-dimensional matrix $\widehat{K}$ are obtained from

$$
K_{l_{1} l_{2}}=\frac{E_{1} E_{2} \exp \left[-\frac{|x|-i y}{2} \frac{u_{1}+u_{2}}{2}-\frac{|x|+i y}{2} \frac{u_{1}-1+u_{2}-1}{2}\right]}{u_{1}+u_{2}},
$$

and $l_{1}, l_{2}=-\infty, \ldots, 0, \ldots, \infty$. 
Let us summarize: correlation functions of the Ising model on the cylinder in the scaling limit

$$
\begin{aligned}
& \left\langle\sigma(0,0) \sigma\left(r_{x}, r_{y}\right)\right\rangle^{(-)}=\xi \widetilde{\xi}_{T}(\beta) \mathrm{e}^{-|x| / \widetilde{\Lambda}(\beta)} \tau_{-}(x, y, \beta), \quad T<T_{c}, \\
& \left\langle\sigma(0,0) \sigma\left(r_{x}, r_{y}\right)\right\rangle^{(+)}=\xi \widetilde{\xi}_{T}(\beta) \mathrm{e}^{-|x| / \widetilde{\Lambda}(\beta)} \tau_{+}(x, y, \beta), \quad T>T_{c},
\end{aligned}
$$

can be expressed through the determinants of infinite-dimensional matrices

$$
\begin{aligned}
& \tau_{-}+\tau_{+}=\left|1+\frac{1}{\beta} \widehat{K}\right|, \\
& \tau_{-}-\tau_{+}=\left|1-\frac{1}{\beta} \widehat{K}\right|,
\end{aligned}
$$

where $\widehat{K}$ is defined by $(2.17)$.

On the infinite lattice $\widehat{K}$ reduces to integral operator with the kernel

$$
K(u, v)=\frac{1}{\pi} \frac{\mathrm{e}^{-\frac{|x|-i y}{2} \frac{u+v}{2}-\frac{|x|+i y}{2} \frac{u^{-1}+v^{-1}}{2}}}{u+v},
$$

acting on $L^{2}(0, \infty)$, and matrix determinants in the right hand side of $(2.20)$ and (2.21) become Fredholm determinants.

\section{Nonlinear differential equations}

In recent years numerous connections between Fredholm determinants of certain integral operators and differential equations become apparent [13, 14, 15]. We will formulate here only necessary results.

Let us consider integral operator $\widehat{K}$ with the kernel

$$
K(x, y)=\frac{E(x) E(y)}{x+y}, \quad E(x)=e(x) \mathrm{e}^{z \frac{x}{2}+\bar{z} \frac{x^{-1}}{2}} .
$$

It was shown in [14] that the functions

$$
\begin{aligned}
& \varphi=\ln |1+\lambda \widehat{K}|-\ln |1-\lambda \widehat{K}|, \\
& \psi=\ln |1+\lambda \widehat{K}|+\ln |1-\lambda \widehat{K}|,
\end{aligned}
$$

satisfy differential equations

$$
\partial_{z \bar{z}} \varphi=\frac{1}{2} \sinh 2 \varphi
$$




$$
\partial_{z \bar{z}} \psi=\frac{1-\cosh 2 \varphi}{2} .
$$

To derive them, one has to define

$$
\begin{aligned}
& u_{i, j}=u_{j, i}=\left\langle E_{i}\left|\frac{1}{1-\widehat{K}^{2}}\right| E_{j}\right\rangle, \\
& v_{i, j}=v_{j, i}=\left\langle E_{i}\left|\frac{\widehat{K}}{1-\widehat{K}^{2}}\right| E_{j}\right\rangle,
\end{aligned}
$$

where $E_{i}(x)=x^{i} E(x)$, and to prove the following recursion relations

$$
\begin{gathered}
u_{i+1, j}-u_{i, j+1}=u_{i, 0} v_{j, 0}-v_{i, 0} u_{j, 0} \\
v_{i+1, j}+v_{i, j+1}=u_{i, 0} u_{j, 0}-v_{i, 0} v_{j, 0}
\end{gathered}
$$

and differentiation formulae

$$
\begin{gathered}
\left\{\begin{array}{c}
\partial_{z} u_{p, q}=\frac{1}{2}\left(u_{p, 0} v_{q, 0}+v_{p, 0} u_{q, 0}\right)+\frac{1}{2}\left(u_{p+1, q}+u_{p, q+1}\right), \\
\partial_{\bar{z}} u_{p, q}=\frac{1}{2}\left(u_{p,-1} v_{q,-1}+v_{p,-1} u_{q,-1}\right)+\frac{1}{2}\left(u_{p-1, q}+u_{p, q-1}\right),
\end{array}\right. \\
\left\{\begin{array}{c}
\partial_{z} v_{p, q}=\frac{1}{2}\left(u_{p, 0} u_{q, 0}+v_{p, 0} v_{q, 0}\right)+\frac{1}{2}\left(v_{p+1, q}+v_{p, q+1}\right), \\
\partial_{\bar{z}} v_{p, q}=\frac{1}{2}\left(u_{p,-1} u_{q,-1}+v_{p,-1} v_{q,-1}\right)+\frac{1}{2}\left(v_{p-1, q}+v_{p, q-1}\right), \\
\partial_{z} \varphi=u_{0,0}, \quad \partial_{\bar{z}} \varphi=u_{-1,-1}, \\
\partial_{z} \psi=-v_{0,0}, \quad \partial_{\bar{z}} \psi=-v_{-1,-1} .
\end{array}\right.
\end{gathered}
$$

Two additional equations

$$
\begin{aligned}
\partial_{z z} \psi & =-\left(\partial_{z} \varphi\right)^{2}, \\
\partial_{\overline{z z}} \psi & =-\left(\partial_{\bar{z}} \varphi\right)^{2},
\end{aligned}
$$

were not observed in [14]. To derive, for example, first of them, one has to differentiate (3.12) with respect to $z$ and to use the recursion relation (3.8).

It can be shown that the recursion relations (3.7), (3.8), differentiation formulae (3.9)-(3.12) and differential equations (3.3), (3.4), (3.13), (3.14) remain valid even when $\widehat{K}$ is a matrix (finite- or infinite-dimensional) with the elements

$$
K_{m n}=\frac{e_{m} e_{n} \mathrm{e}^{z \frac{x_{m}+x_{n}}{2}+\bar{z} \frac{x_{m}-1}{2}+x_{n}-1}}{x_{m}+x_{n}} .
$$

The matrix (2.17) belongs to this class, so after denoting

$$
\varphi=\ln \frac{\tau_{-}+\tau_{+}}{\tau_{-}-\tau_{+}}, \quad \psi=\ln \left(\tau_{-}^{2}-\tau_{+}^{2}\right),
$$




$$
z=-\frac{|x|-i y}{2}, \quad \bar{z}=-\frac{|x|+i y}{2},
$$

we can write down a system of equations for the correlation functions

$$
\begin{gathered}
\tau_{-}=\mathrm{e}^{\frac{\psi}{2}} \cosh \frac{\varphi}{2}, \\
\tau_{+}=\mathrm{e}^{\frac{\psi}{2}} \sinh \frac{\varphi}{2}, \\
\partial_{z \bar{z}} \varphi=\frac{1}{2} \sinh 2 \varphi, \\
\partial_{z \bar{z}} \psi=\frac{1-\cosh 2 \varphi}{2}, \\
\partial_{z z} \psi=-\left(\partial_{z} \varphi\right)^{2}, \\
\partial_{\overline{z z}} \psi=-\left(\partial_{\bar{z}} \varphi\right)^{2} .
\end{gathered}
$$

On the infinite lattice in the scaling limit correlation function and, therefore, the functions $\varphi$ and $\psi$ become invariant under rotations in the $(x, y)$ plane. In this case, one can define $\theta=2(z \bar{z})^{\frac{1}{2}}, \eta(\theta)=\mathrm{e}^{-\varphi(2 z, 2 \bar{z})}$ and then sinh-Gordon equation (3.19) reduces to Painlevé III equation (1.6) for the function $\eta(\theta)$.

Before proceed with the differential equations, let us discuss the boundary conditions for (3.19). It should be noted that the asymptotical conditions at $|x| \rightarrow \infty$ in the case of the cylinder do not determine the solution uniquely. Indeed, the asymptotics of the functions $\tau_{ \pm}(x, y)$ is determined by the first terms of the corresponding form factor expansions, and moreover, in the expression for $g_{1}(2.13)$ we should take $l=0$ (this is not the case for the infinite lattice, where the sum over $l$ transforms into an integral and gives the modified Bessel function). Therefore, we obtain

$$
\tau_{+} \rightarrow \frac{1}{\beta} \mathrm{e}^{-|x|-\widetilde{\nu}(0, \beta)}, \quad \tau_{-} \rightarrow 1
$$

as $|x| \rightarrow \infty$. The corresponding asymptotics of $\varphi$

$$
\varphi \rightarrow \frac{2}{\beta} \mathrm{e}^{-|x|-\widetilde{\nu}(0, \beta)}
$$

does not depend on $y$-coordinate. If we assume that the last condition defines the solution uniquely, it would not depend on $y$ for all values of $x$ and obviously, this is not true.

If it were possible to obtain an additional differential equation, containing the derivatives with respect to $\frac{1}{\beta}$ (this quantity corresponds to QFT temperature), one would be able to use the solution of Painlevé III equation 
(1.6) with the boundary conditions (1.7) as the initial condition at $\frac{1}{\beta}=0$. However, the derivation of such equation stands as an open problem. This problem is rather difficult to solve, mainly due to the complexity of the function $\widetilde{\nu}(l, \beta)$.

Therefore, we have no way other than the classical one - to determine, at least numerically, the function $\varphi(x, y)$ and its derivative on the whole line $(y=$ const $)$ or on the circle $(x=$ const $)$. Such boundary conditions, combined with the periodicity in $y$-coordinate, will completely determine the solution of sinh-Gordon equation (3.19).

Now we can turn to the derivation of the equations for the functions $\tau_{ \pm}$. If one denotes

$$
u=\ln \tau_{-},
$$

then by means of usual differentiation and using of relations (3.17)-(3.22) it is straightforward to obtain the following identities

$$
\begin{gathered}
u_{z z}=\frac{\cosh \varphi-1}{2} \partial_{z}\left(\frac{\partial_{z} \varphi}{\sinh \varphi}\right) \\
u_{\overline{z z}}=\frac{\cosh \varphi-1}{2} \partial_{\bar{z}}\left(\frac{\partial_{\bar{z}} \varphi}{\sinh \varphi}\right) \\
u_{z \bar{z}}=\frac{\cosh \varphi-1}{2}\left[-1+\left(\frac{\partial_{z} \varphi}{\sinh \varphi}\right)\left(\frac{\partial_{\bar{z}} \varphi}{\sinh \varphi}\right)\right] \\
u_{z z \bar{z}}=u_{z \bar{z}}\left(\frac{\partial_{z} \varphi}{\sinh \varphi}\right)+u_{z z}\left(\frac{\partial_{\bar{z}} \varphi}{\sinh \varphi}\right) \\
u_{z \overline{z z}}=u_{\overline{z z}}\left(\frac{\partial_{z} \varphi}{\sinh \varphi}\right)+u_{z \bar{z}}\left(\frac{\partial_{\bar{z}} \varphi}{\sinh \varphi}\right) \\
u_{z z \overline{z z}}=u_{z \overline{z z}}\left(\frac{\partial_{z} \varphi}{\sinh \varphi}\right)+u_{z z \bar{z}}\left(\frac{\partial_{\bar{z}} \varphi}{\sinh \varphi}\right)-2 u_{z \bar{z}}^{2}+\frac{2\left[u_{z z} u_{\overline{z z}}-u_{z \bar{z}}^{2}\right]}{\cosh \varphi-1} .
\end{gathered}
$$

The last four relations can be treated as a system of algebraic equations for three variables $\frac{\cosh \varphi-1}{2},\left(\frac{\partial_{z} \varphi}{\sinh \varphi}\right),\left(\frac{\partial_{\bar{z}} \varphi}{\sinh \varphi}\right)$. The equation for correlation function

$$
u_{z \bar{z}}\left(u_{z z \overline{z z}}+2 u_{z \bar{z}}^{2}-u_{z \bar{z}}\right)=u_{z z \bar{z}} u_{z \overline{z z}}-u_{z z} u_{\overline{z z}}
$$

appears as the consistency condition for this system. In the case $u=\ln \tau_{+}$all the calculations can be performed in analogous fashion, and, though equations (3.23)-(3.28) change their form, the equation (3.29) for the correlation function is the same. Since (3.29) is obtained from (integrable) sinh-Gordon equation (3.19) and three linear equations (3.20)-(3.22), it is very likely integrable. More detailed investigation of (3.29) is beyond the scope of this paper. 
On the infinite lattice the problem of derivation of differential equations for the correlation functions is much simpler due to rotational invariance. The functions $\tau_{ \pm}, \varphi, \psi, u$ depend on the single variable $t=2(z \bar{z})^{\frac{1}{2}}$. Instead of (3.19) one has

$$
\varphi^{\prime \prime}+\frac{1}{t} \varphi^{\prime}=\sinh \varphi \cosh \varphi,
$$

and instead of (3.23), (3.25), (3.26) -

$$
\begin{gathered}
u^{\prime \prime}-\frac{1}{t} u^{\prime}=\frac{\left(\varphi^{\prime \prime}-\frac{1}{t} \varphi^{\prime}\right) \sinh \varphi-\left(\varphi^{\prime}\right)^{2} \cosh \varphi}{2(\cosh \varphi+1)}, \\
u^{\prime \prime}+\frac{1}{t} u^{\prime}=\frac{\cosh \varphi-1}{2}\left[-1+\left(\frac{\varphi^{\prime}}{\sinh \varphi}\right)^{2}\right], \\
\left(u^{\prime \prime}+\frac{1}{t} u^{\prime}\right)^{\prime}=2 u^{\prime \prime}\left(\frac{\varphi^{\prime}}{\sinh \varphi}\right),
\end{gathered}
$$

correspondingly. When we again regard the last four relations as a system for three variables $\varphi, \varphi^{\prime}, \varphi^{\prime \prime}$ and if we denote

$$
\zeta=t u^{\prime}
$$

we will obtain well-known result

$$
\left(t \zeta^{\prime \prime}\right)^{2}=4\left(t \zeta^{\prime}-\zeta\right)^{2}-4\left(\zeta^{\prime}\right)^{2}\left(t \zeta^{\prime}-\zeta\right)+\left(\zeta^{\prime}\right)^{2}
$$

- the equation for the $\tau$-function of the Painlevé $\mathrm{V}$ equation, - as the consistency condition for this system. Analogous calculations lead to the same equation for $\tau_{+}$.

I am grateful to V. N. Shadura for suggesting the problem and constant help, to V. N. Rubtsov and especially to A. I. Bugrij - for fruitful discussions and critical comments. This work was supported by the INTAS program under grant INTAS-00-00055 and by Ukrainian SFFR project No. $02.07 / 00152$.

\section{References}

[1] T. T. Wu, B. M. McCoy, C. A. Tracy, and E. Barouch, "Spin-spin correlation functions for the two-dimensional Ising model: Exact theory in the scaling region", Phys. Rev. B13 (1976) 316. 
[2] B. M. McCoy, C. A. Tracy, and T. T. Wu, "Painlevé functions of the third kind", J. Math. Phys. 18 (1977) 1058.

[3] B. M. McCoy, J. H. H. Perk, "Relation of conformal field theory and deformation theory for the Ising model", Nucl. Phys. B285 (1987) 279.

[4] M. Jimbo, T. Miwa, Y. Mori, and M. Sato, "Density matrix of an impenetrable Bose gas and the fifth Painlevé transcendent", Physica D (1980) 80.

[5] J. H. H. Perk, "Equations of motion for the transverse correlations of the one dimensional XY model at finite temperature", Phys. Letts. A79 (1980) 1.

[6] F. H. L. Essler, H. Frahm, A. R. Its, and V. E. Korepin, "Integrodifference equation for a correlation function of the spin- $\frac{1}{2}$ Heisenberg XXZ chain", Nucl. Phys. B446 (1995) 448; cond-mat/9503142.

[7] D. Bernard, A. LeClair, "Differential equations for sine-Gordon correlation functions at the free fermion point", Nucl. Phys. B426 (1994) 534.

[8] A. I. Bugrij, "Correlation function of the two-dimensional Ising model on the finite lattice. I", Theor. \& Math. Phys. 127 (2001) 143.

[9] A. I. Bugrij, O. O. Lisovyy, "Magnetic susceptibility of the twodimensional Ising model on the finite lattice", Zh. Eksp. Teor. Fiz. 121 (2002) 1; hep-th/0106270.

[10] J. Palmer, C. A. Tracy, "Two-dimensional Ising correlations: convergence of the scaling limit", Advances in Applied Mathematics. V. 2 (1981) 329 .

[11] K. Yamada, "On the spin-spin correlation function in the Ising square lattice and the zero field susceptibility", Prog. Theor. Phys. 71 (1984) 1416.

[12] A. I. Bugrij, "Form factor representation of the correlation function of the two dimensional Ising model on a cylinder", in Integrable Structures of Exactly Solvable Two-Dimensional Models of Quantum Field Theory, eds. S. Pakuliak and G. von Gehlen, Kluwer Academic Publ. NATO Series; hep-th/0107117.

[13] C. A. Tracy, H. Widom, "Fredholm determinants, differential equations and matrix models", Commun. Math. Phys. 163 (1994) 33. 
[14] C. A. Tracy, H. Widom, "Fredholm determinants and the $\mathrm{mKdV} /$ sinhGordon hierarchies", Commun. Math. Phys. 179 (1996) 1.

[15] H. Widom, "On the solution of a Painlevé III equation", Math. Physics, Analysis and Geometry, 3 (2000) 375. 